\title{
CERTAIN C*-ALGEBRAS WITH NON-ZERO REAL RANK AND EXTREMAL RICHNESS
}

\author{
HIROYUKI OSAKA
}

\begin{abstract}
We know that real rank zero is not stable under the spatial $\mathrm{C}^{*}$-algebraic tensor product. Indeed, if $H$ is a separable infinite dimensional Hilbert space, we show that $B(H) \otimes B(H)$ has not real rank zero, although $B(H)$ has real rank zero. We can also construct many interesting $\mathrm{C}^{*}$-algebras with non-zero real rank. To determine their real rank, we investigate the size of one-sided invertible elements $V(A)$ in a $\mathrm{C}^{*}$-algebra $A$, and we show that $\overline{V(A)}=A$ under some conditions. From this observation we know that these $C^{*}$-algebras are extremally rich, which is defined by Brown and Pedersen ([3]). In particular, their real rank is at most one.
\end{abstract}

\section{Introduction}

Let $A$ be a unital $\mathrm{C}^{*}$-algebra and $A_{\mathrm{sa}}$ be the set of self-adjoint elements. The concept of the real rank of a $\mathrm{C}^{*}$-algebra $A(=\mathrm{RR}(A))$ was defined by Brown and Pedersen ([2]) as follows: $\operatorname{RR}(A)$ is the least integer $n$ such that $\left\{\left(a_{0}, a_{1}, \cdots, a_{n}\right) \in A_{\mathrm{sa}}^{n+1}: \sum_{i=0}^{n} A a_{i}=A\right\}$ is dense in $A_{\mathrm{sa}}^{n+1}$. If $A$ is non-unital, its real rank is defined by $\operatorname{RR}(\tilde{A})$, where $\tilde{A}$ is the $\mathrm{C}^{*}$-algebra obtained by adding a unit to $A$.

An important part of the motivation for introducing it is to have an analogue of the dimension concept for $\mathrm{C}^{*}$-algebras. In the commutative case, if $X$ is a compact Hausdorff space, then $\operatorname{dim} X$ can be defined as a property of the algebra $C(X)$ of continuous functions on $X$. Thus, $\operatorname{dim} X \leq n$ if every continuous function $f: X \rightarrow \mathscr{R}^{n+1}$ can be approximated by another continuous function $g$, for which $0 \notin g(X)$ ([23]). If $X$ is compact Hausdorff space, then $\operatorname{RR}(C(X))=\operatorname{dim} X$.

We hope that the real rank would have the parallel property of the dimension theory such that $\operatorname{dim}(X \times Y) \leq \operatorname{dim} X+\operatorname{dim} Y$. However, Kodaka and the author ([14] [15]) showed that $\mathrm{RR}(A \otimes B) \leq \mathrm{RR}(A)+\mathrm{RR}(B)$ does not hold even if both $C^{*}$-algebras are separable, nuclear and have real rank zero, where $\otimes$ means the spatial $\mathrm{C}^{*}$-algebraic tensor product. For example, $E_{n} \otimes O_{n} \otimes O_{n}(n \geq 3)$ does not have real rank zero, although $E_{n}$ and $O_{n} \otimes O_{n}$

Received January 8, 1996; in revised form November 30, 1998 and December 15, 1998. 
have real rank zero ([15]), where $O_{n}$ is the Cuntz algebra generated by nelements $S_{1}, S_{2}, \cdots, S_{n}$ such that $S_{i}^{*} S_{j}=\delta_{i, j}(1 \leq i, j \leq n)$ and $\sum_{i=1}^{n} S_{i} S_{i}^{*}=1$, and $E_{n}$ is the $\mathrm{C}^{*}$-subalgebra generated by $S_{1}, S_{2}, \cdots, S_{n}$ of $O_{n+1}$ ([5]). We note that if $A \otimes B$ has not real rank zero, then any $\mathrm{C}^{*}$-algebraic tensor product of $A$ and $B$ has not real rank zero.

The motivation of this paper is to determine the real rank of $E_{n} \otimes O_{n} \otimes O_{n}$.

Before calculating its real rank, we must touch on recent works due to Pedersen ([25]) and Brown and Pedersen ([3] [4]). Pedersen tried to describe the geometry of the unit ball of a $\mathrm{C}^{*}$-algebra from the concept of the $\lambda$ property in operator algebras. Here, a $\mathrm{C}^{*}$-algebra $A$ has the $\lambda$-property if any element $T$ in the closed unit ball $A_{1}$ of $A, \lambda(T)=\sup \{\lambda \mid T=$ $\left.\lambda V+(1-\lambda) B ; V \in \operatorname{ex}\left(A_{1}\right), B \in A_{1}, \lambda \in[0,1]\right\}$ is positive, where $\operatorname{ex}\left(A_{1}\right)$ means the set of extremal points in $A_{1}$. He showed that every von Neumann algebra has the $\lambda$-property and that a prime $\mathrm{C}^{*}$-algebra $A$ has the $\lambda$-property if and only if the set of one-sided invertible elments $V(A)$ is dense in $A$. In particular, purely infinite simple $C^{*}$-algebra(not necessarily unital) has $\lambda$ property. Related to the real rank, he proved that the real rank of $\mathrm{C}^{*}$-algebras with the $\lambda$-property is at most one. After that, he and Brown succeeded this work and clarified the structure of the gometry of the unit ball of a $\mathrm{C}^{*}$ algebra $\mathrm{A}$ with the $\lambda$-property, i.e., $A$ has the $\lambda$-property if and only if the set of quasi-invertible elements $A^{-1} \operatorname{ex}\left(A_{1}\right) A^{-1}$ is dense in $A$ if and only if for every $T$ in $A_{1}$ and $0<\epsilon<\frac{1}{2}$, there are extremal points $U_{1}, U_{2}, U_{3}$ in $\operatorname{ex}\left(A_{1}\right)$ such that $T=\frac{1}{2}(1-\epsilon) U_{1}+\frac{1}{2}(1-\epsilon) U_{2}+\epsilon U_{3}$ ([4] [25]). They say that a unital $\mathrm{C}^{*}$-algebra $A$ is "extremally rich" if $A$ satisfies the $\lambda$-property. A non-unital $\mathrm{C}^{*}$-algebra $A$ then is called extremally rich if its unitaization $\tilde{A}$ is extremally rich.

We also note that $\mathrm{R} \varnothing \mathrm{rdam}$ ([30]) investigated the size of $V(A)$ and his method is very useful to prove the extremal richness of some $\mathrm{C}^{*}$-algebras.

In section 1 , we present examples of $C^{*}$-algebras with non-zero real rank. In particular, we show that $B(H) \otimes B(H)$ has not real rank zero. After that we consider an extension $E$ of $A$ by $B$, and show that $E$ is extremally rich if both $A$ and $B$ are purely infinite simple $\mathrm{C}^{*}$-algebras. From this observation we know that $E_{n} \otimes O_{n} \otimes O_{n}(n \geq 3)$ has real rank one. At the last section we note that $B(H) \otimes B(H)$ is not extremally rich. So, we don't know its real rank yet.

\section{1. $C^{*}$-algebras with non-zero real rank}

As Zhang pointed out in [35] (cf. [2]), even if $E$ is an extension of $A$ by $B$ and both $A$ and $B$ have real rank zero, we can not in general deduce that $E$ has 
real rank zero. This obstruction follows from the lifting problem of projections. He proved ([35, Proposition 2.3]) that if $K_{1}(B)=0$, then any projection in $A$ can be lifted to a projection in $E$, and $E$ has real rank zero. From this observation we get the following result which is an extended version of the result in [14].

Note that by $A \otimes B$ we denote the spatial $C^{*}$-algebraic tensor product of $A$ and $B$.

Proposition 1.1. Let $A$ be a unital $C^{*}$-algebra with non-trivial $K_{1}$-group $\left(K_{1}(A) \neq 0\right)$ and let $H$ be a separable infinite dimensional Hilbert space. Then, $\mathrm{RR}(A \otimes B(H)) \neq 0$.

Proof. Consider the following $\mathrm{C}^{*}$-exact sequence:

$$
0 \longrightarrow A \otimes \mathscr{K} \longrightarrow A \otimes B(H) \stackrel{\pi}{\longrightarrow} A \otimes B(H) / A \otimes \mathscr{K} \longrightarrow 0,
$$

where $\mathscr{K}$ denotes the $\mathrm{C}^{*}$-algebra of all compact operators on $H$.

Since $K_{i}(A \otimes B(H))=0(i=1,2)([33$, Theorem 10.2]) we have

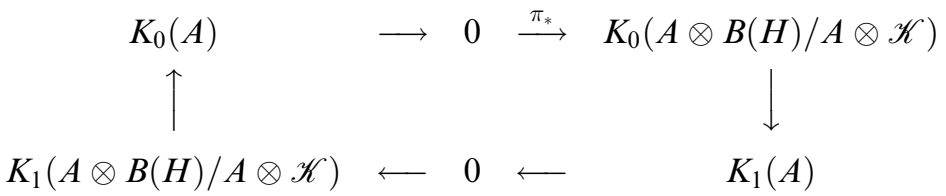

by the sixterm exact sequence ([33, Theorem 9.3.2]).

We know that $\pi_{*}$ is not surjective. Hence, there is a projection in $(A \otimes B(H) / A \otimes \mathscr{K}) \otimes \mathscr{K}$ which can not be lifted to a projection in $A \otimes B(H) \otimes \mathscr{K}$. So, $\mathrm{RR}(A \otimes B(H) \otimes \mathscr{K}) \neq 0$, and $\mathrm{RR}(A \otimes B(H)) \neq 0$ by $[2$, Theorem 3.14] [35, Theorem 3.2].

Corollary 1.2. Let $H$ be a separable infinite dimensional Hilbert space. Then $\operatorname{RR}(B(H) \otimes B(H)) \neq 0$.

Proof. Let $Q(H)$ be the Calkin algebra $B(H) / \mathscr{K}$. Since $K_{1}(Q(H)) \cong Z$, we know that $\operatorname{RR}(Q(H) \otimes B(H)) \neq 0$, hence $\operatorname{RR}(B(H) \otimes B(H)) \neq 0$ from the property of real rank zero to pass to quotients.

REMARK 1.3. (1) We can also construct a separable $\mathrm{C}^{*}$-algebra $A \otimes B$ with non-zero real rank, although both $A$ and $B$ have real rank zero ([15]):

Let $A$ be a unital simple $C^{*}$-algebra. Suppose that $K_{1}(A)$ contains a finite group $\mathrm{Z}_{n}$ as a summand. Then, $\mathrm{RR}\left(A \otimes E_{n}\right) \neq 0$ if $n \geq 3$. For examples, take $O_{n} \otimes O_{n}$ and $C_{\gamma}^{*}\left(F_{k}\right) \otimes O_{n}$ for $A$, where $C_{\gamma}^{*}\left(F_{k}\right)$ is the reduced group C*-algebra of the free group with $k$ generators.

(2) Let $A$ be a $\sigma$-unital purely infinite simple $\mathrm{C}^{*}$-algebra. Then, Zhang ([38, Theorem 2.1]) proved that $M(A)$ has real rank zero if and only if 
$K_{1}(A)=0$. Moreover, we can easily construct purely infinite simple $\mathrm{C}^{*}$-algebras with non-trivial $K_{1}$-group from the fact that $A \otimes O_{\infty}$ is purely infinite simple $\mathrm{C}^{*}$-algebra if $A$ is simple ([13]), where $O_{\infty}$ is a $\mathrm{C}^{*}$-algebra generated by countably many orthogonal isometries on some Hilbert space ([5]).

\section{Extremal richness and Real rank}

In this section, we consider a $\mathrm{C}^{*}$-exact sequence $0 \rightarrow I \rightarrow A \rightarrow A / I \rightarrow 0$ in case that $I$ is an essential closed two-sided ideal, and both $I$ and $A / I$ are purely infinite simple $C^{*}$-algebras. Using the idea in [30] we can conclude that $A$ is extremally rich.

At first we introduce Rørdam's work. Let $A$ be a unital $\mathrm{C}^{*}$-algebra and $\mathrm{ZD}(A)$ be the set of elements in $A$ for which $a x=0=x b$ for some non-zero elements $a$ and $b$ in $A$. In [30] he noted that the closure of $\operatorname{ZD}(A)(=\overline{\operatorname{ZD}(A)})$ is the set of all elements in $A$ that are not one-sided invertible. Therefore, if we could prove that $\mathrm{ZD}(A)$ is contained in the closure of the set of all onesided invertible elements in $A(=V(A))$, then we know that $V(A)$ is dense in $A$, and hence, $A$ is extremally rich ([25, Theorem 8.3]). He proved that a purely infinite simple unital $\mathrm{C}^{*}$-algebra $A$ satisfies $\mathrm{ZD}(A) \subseteq \overline{\mathrm{GL}(A)}$, where $\mathrm{GL}(A)$ denotes the set of all invertible elements in $A$.

Using this concept we get the main result which is an extended version of [30, Theorem 4.5]. The proof of the following theorem is omitted because the proof in [17, Corollary 3.7] is better than our original proof. We note that our result has the priority over [17].

Theorem 2.1. Let $A$ be a unital $C^{*}$-algebra and $J$ be an essential closed twosided ideal of $A$. Suppose that $J$ and $A / J$ are purely infinite simple $C^{*}$-algebras. Then $\mathrm{ZD}(A) \subset \overline{V(A)}$. In particular, $A$ is extremally rich and $\mathrm{R} \operatorname{R}(A) \leq 1$.

Remark 2.2. Combining Theorem 10.4 of [25] with Theorem 3.7 of [4] we know that every extremally rich $\mathrm{C}^{*}$-algebra has real rank at most one. We note that its direct proof is appeared in Proposition 3.6 of [12].

Corollary 2.3. Let $A$ be a unital, exact, separable, and purely infinite simple $C^{*}$-algebra. Then $A \otimes B(H)$ is extremally rich and

$$
\mathrm{RR}(A \otimes B(H))=\left\{\begin{array}{l}
0 \text { if } K_{1}(A)=0 \\
1 \text { if } K_{1}(A) \neq 0 .
\end{array}\right.
$$

Proof. Consider the following $\mathrm{C}^{*}$-exact sequence:

$$
0 \longrightarrow A \otimes \mathscr{K} \longrightarrow A \otimes B(H) \longrightarrow A \otimes Q(H) \longrightarrow 0 .
$$

Note that $A \otimes Q(H)$ is a purely infinite simple $\mathrm{C}^{*}$-algebra by [13]. 
The first assertion comes from the previous theorem.

The second assertion comes from Proposition 1.1, [2, Theorem 3.14], [35, Theorem 3.2], and the previous theorem.

Corollary 2.4. Let A be a $\sigma$-unital, purely infinite simple $C^{*}$-algebra. Then $M(A)$ is extremally rich, and

$$
\operatorname{RR}(M(A))=1 \quad \text { if } K_{1}(A) \neq 0 \text { and } 1 \notin A
$$

Proof. Consider the following $\mathrm{C}^{*}$-exact sequence:

$$
0 \longrightarrow A \longrightarrow M(A) \longrightarrow M(A) / A \longrightarrow 0 .
$$

Since $A$ is stable ([38, Theorem 1.2]) and $M(A) / A$ is a purely infinite simple $\mathrm{C}^{*}$-algebra ([29, Theorem 3.2][34, Theorem 3.3]) we can get the assertion through the same argument as in Corollary 2.3.

Corollary 2.5. Let $n$ be an integer more than or equal to 2 and $A$ be a unital purely infinite simple $C^{*}$-algebra. Then $A \otimes E_{n}$ is extremally rich. Moreover, $\operatorname{RR}\left(A \otimes E_{n}\right) \leq 1$.

Proof. Consider the following $\mathrm{C}^{*}$-exact sequence ([8]):

$$
0 \longrightarrow A \otimes \mathscr{K} \longrightarrow A \otimes E_{n} \longrightarrow A \otimes O_{n} \longrightarrow 0 .
$$

Since $A \otimes O_{n}$ is a purely infinite simple $\mathrm{C}^{*}$-algebra ([11] [13] [26] [31]) we get the assertion from Theorem 2.1.

ReMark 2.6. (1) We can not replace a pure infiniteness of $J$ by $\mathrm{RR}(J)=0$. Indeed, when $J$ is a finite matroid algebra, we know that $M(J)$ is not extremally rich ([3] [17])

(2) In Corollary 2.4 , if $A$ has no unit and has trivial $K_{1}$-group, Zhang proved ([38, Theorem 2.1]) that $\mathrm{RR}(M(A))=0$.

(3) From Corollary 2.5, we know that both $\mathrm{C}^{*}$-algebras $O_{n} \otimes O_{n} \otimes E_{n}$ and $C_{\gamma}^{*}\left(F_{k}\right) \otimes O_{n} \otimes E_{n}$ have real rank one if $n \geq 3$ (when $n=2$ they have real rank zero).

\section{Non-extremal richness of $B(H) \otimes B(H)$}

Since $B(H) \otimes B(H)$ is a prime $\mathrm{C}^{*}$-algebra and $B(H)$ is extremally rich, we might hope that it is extremally rich. However, we know that it is false.

As the same in the case of real rank (see §1) an obstruction to have the extremal richness is a lifting problem of extremal points in the closed unit ball of a quotient $\mathrm{C}^{*}$-algebra of some $\mathrm{C}^{*}$-subalgebra of $B(H) \otimes B(H)$ as follows.

Let $B \quad$ be a closed two-sided ideal $B(H) \otimes \mathscr{K}+\mathscr{K} \otimes B(H)$ of $B(H) \otimes B(H)$, and let $D$ be a $C^{*}$-algebra $Q(H) \otimes \mathscr{K} \oplus \mathscr{K} \otimes Q(H)$, where 
$Q(H)$ is the Calkin algebra $B(H) / \mathscr{K}$. Then, we have the following $\mathrm{C}^{*}$-exact sequence:

$$
0 \longrightarrow \mathscr{K} \otimes \mathscr{K} \longrightarrow B \longrightarrow D \longrightarrow 0 \text {. }
$$

Recall that $\tilde{A}$ denotes the unitaization of a $\mathrm{C}^{*}$-algebra $A$.

Lemma 3.1. Let $H$ be a separable infinite dimensional Hilbert space and $\mathscr{K}$ be the $C^{*}$-algebra generated by compact operators on $H$. Then, there is an extremal element in the closed unit ball of $\tilde{D}$ which can not be lifted to an extremal element in the closed unit ball of $\tilde{B}$.

Proof. Note that we have the following $C^{*}$-exact sequence:

$$
0 \longrightarrow \mathscr{K} \otimes \mathscr{K} \longrightarrow \tilde{B} \longrightarrow \tilde{D} \longrightarrow 0 .
$$

Since $\tilde{B}$ is prime, any extremal element in the closed unit ball of $\tilde{B}$ is either an isometry or a co-isometry. Therefore, we have only to show that there is an extremal element in the closed unit ball of $\tilde{D}$ that is neither an isometry nor a co-isometry.

Since $Q(H) \otimes \mathscr{K}$ is purely infinite simple, there is a partial isometry $v \in Q(H) \otimes \mathscr{K}$ such that $v^{*} v=p$ and $v v^{*}<p$. Similarly, there is a partial isometry $u \in \mathscr{K} \otimes Q(H)$ such that $u u^{*}=q$ and $u^{*} u<q$. Set $w=(v, u)+$ $1-(p, q)$. Then, $w^{*} w=1-(p, q)+\left(p, u^{*} u\right)$ and $w w^{*}=1-(p, q)+\left(v v^{*}, q\right)$. So, $w$ is a partial isometry, but $w$ is neither an isometry nor a co-isometry. Moreover, $\left(1-w^{*} w\right) \tilde{D}\left(1-w w^{*}\right)=\{0\}$. Hence $w$ is an extremal element by $[24,1.4 .7]$, and this is required one.

Proposition 3.2. Under the same assumption as in Lemma $3.1 B(H) \otimes$ $B(H)$ is not extremally rich.

Proof. From the previous lemma we know that $\tilde{B}$ is not extremally rich ([3, Theorem 6.1]). Hence, since $B$ is a closed two-sided ideal in $B(H) \otimes B(H), B(H) \otimes B(H)$ is not extremally rich by [3, Theorem 3.5].

Question 3.3.

Is real rank of $B(H) \otimes B(H)$ one ?

ACKNOWLEDGements. This work was done while the author visited The Fields Institute for Mathematical Research for the program year (Sept., 1994-Aug., 1995) in Operator Algebras and its Applications.

It is a pleasure to thank Gert K. Pedersen for bringing the "extremal richness" and its literature to my attention. Thanks also to Rajarama Bhat and Nadia Larsen for valuable discussions and suggestions.

The author would like to thank George A. Elliott for his kind arrangement for this visit. 


\section{REFERENCES}

1. B. Blackadar, A. Kumjian and M. Rørdam, Approximation central matrix units and the structure of non-commutative tori, K-theory 6 (1992), 267-284.

2. L. G. Brown and G. K. Pedersen, $C^{*}$-algebras of real rank zero, J. Funct. Anal. 99 (1991), $131-149$.

3. L. G. Brown and G. K. Pedersen, On the geometry of the unit ball of a $C^{*}$-algebra, J. reine angew. Math. 469 (1995), 113-147.

4. L. G. Brown and G. K. Pedersen, Approximation and convex decomposition by extremals in a $C^{*}$-algebra, Math. Scand. 81 (1997), 69-85.

5. J. Cuntz, Simple $C^{*}$-algebras generated by isometries, Comm. Math. Phys. 57 (1977), 173185.

6. J. Cuntz, The structure of multiplication and addition in simple $C^{*}$-algebras, Math. Scand. 40 (1977), 215-233.

7. J. Cuntz, K-theory for certain $C^{*}$-algebras, Ann. of Math. 113 (1981), 181-197.

8. J. Cuntz, K-theory for certain $C^{*}$-algebras II, J. Operator Theory 5 (1981), 101-108.

9. G. A. Elliott, Derivations of matroid $C^{*}$-algebras, II, Ann. of Math. 100 (1974), 407-422.

10. G. A. Elliott, On the classification of $C^{*}$-algebras of real rank zero, J. Reine. Angew. Math. 443 (1993), 179-219.

11. J. A Jeong, K. Kodaka and H. Osaka, Purely infinite simple crossed products II, Canadian Math. Bull. 39 (1996), 203-210.

12. J. A Jeong and H. Osaka, Extremally rich $C^{*}$-crossed products and the cancellation property, J. Austral. Math. Soc. Ser. A 64 (1998), 285-301.

13. E. Kirchberg, The classification of purely infinite $C^{*}$-algebras using Kasparov's theory, draft.

14. K. Kodaka and H. Osaka, Real rank of tensor products of $C^{*}$-algebras, Proc. Amer. Math. Soc. 123 (1995), 2213-2215.

15. K. Kodaka and H. Osaka, FS-property for $C^{*}$-algebras, submitted.

16. N. Larsen, Convex structure of the unit ball of a $C^{*}$-algebra, M.Sc. Thesis, Copenghagen University, 1994.

17. N. S. Larsen and H. Osaka, Extremal richness of multiplier algebras and corona algebras of simple $C^{*}$-algebras, J. Operator Theory 38 (1997), 131-149.

18. H. Lin, Generalized Weyl-von Neumann Theorems, Internat. J. Math. 2 (1991), 725-739.

19. H. Lin, Exponential rank of $C^{*}$-algebras with real rank zero and the Brown-Pedersen Conjectures, J. Funct. Anal. 114 (1993), 1-11.

20. H. Lin and H. Osaka, Real rank of multiplier algebras of $C^{*}$-algebras of real rank zero, Operator algebras and their applications (Waterloo ON, 1994/1995), 235-241, Fields Inst., Commun 13, Amer. Math. Soc., Providence RI, 1997.

21. H. Lin and N. C. Phillips, Approximate unitary equivalence of of homomorphisms from $O_{\infty}$, J. Reine Angew. Math. 464 (1995), 173-186.

22. H. Osaka, Counterexamples of Brown-Pedersen's conjectures in " $C^{*}$-algebras of real rank zero", Proc. Amer. Math. Soc. 118 (1993), 495-497.

23. A. R. Pears, Dimensin Theory of General Topology, 1975, Cambrige University Press, London/New York.

24. G. K. Pedersen, $C^{*}$-algebras and their automorphism groups, 1979, Academic Press, London.

25. G. K. Pedersen, The $\lambda$-function in operator algebras, J. Operator Theory 26 (1991), 345-381.

26. N. C. Phillips, Approximate unitary equivalence of homomorphisms from odd Cuntz algerbas, Operator algebras and their applications (Waterloo ON, 1994/1995), 243-255, Fields Inst., Commun 13, Amer. Math. Soc., Providence RI, 1997.

27. M. A. Rieffel, Dimension and stable rank in the K-theory of $C^{*}$-algebras, Proc. London Math. Soc. 47 (1983), 285-302.

28. M. Rørdam, Advances in the theory of unitary rank and regular approximation, Ann. of Math. 128 (1988), 153-172.

29. M. Rørdam, Ideals in the multiplier algebras of stable $C^{*}$-algebras, J. Operator Theory 25 (1991), 283-298. 
30. M. Rørdam, On the structure of simple $C^{*}$-algebras tensored with a UHF-algebra, J. Funct. Anal. 100 (1991), 1-17.

31. M. Rørdam, Classification of inductive limits of Cuntz algebras, J. Reine. Angew. Math. 440 (1993), 175-200.

32. S. Wassermann, A pathology in the ideal space of $L(H) \otimes L(H)$, Indiana Univ. Math. J. 27 (1978), 1012-1020.

33. N. E. Wegge-Olsen, K-Theory and $C^{*}$-algebras, 1993, Oxford University press, Oxford.

34. S. Zhang, A Riesz decomposition property and ideal structure of multiplier algebras, J. Operator Theory 24(1990), 209-226.

35. S. Zhang, $C^{*}$-algebras with real rank zero and internal structure of their corona and multiplier algebras, Part III, Canad. J. Math. 42 (1990), 159-190.

36. S. Zhang, A property of purely infinite simple $C^{*}$-algebras, Proc. Amer. Math. Soc. 109 (1990), 717-720.

37. S. Zhang, $K_{1}$-groups, quasidiagonality, and interpolation by multiplier projections, Trans. Amer. Math. Soc. 325 (1991), 793-818.

38. S. Zhang, Certain $C^{*}$-algebras with real rank zero and their corona and multiplier algebras, Part I, Pacific. J. Math. 155 (1992), 169-197.

MATHEMATICS INSTITUTE

UNIVERSITY OF COPENHAGEN

UNIVERSITESPARKEN 5

DK-2100, COPENHAGEN $\varnothing$

DENMARK
CURRENT ADDRESS

DEPARTMENT OF MATHEMATICS

RITSUMEIKAN UNIVERSITY

KUSATSU, SHIGA, 525-8577

JAPAN 